\title{
Establishing a National Higher Education Accreditation Framework: Challenges and Opportunities in Zambia
}

\author{
Stephen Simukanga ${ }^{\mathrm{a},{ }^{*}}$ and W. James Jacob ${ }^{\mathrm{b},+}$ \\ ${ }^{a}$ Higher Education Authority, Zambia \\ ${ }^{\mathrm{b}}$ University of Pittsburgh, USA
}

\begin{abstract}
This paper addresses the context in which a national accreditation framework is being established in Zambia. It also outlines the role the Higher Education Authority plays in coordinating this national effort. This paper is informed by two primary data sources. The first is a review of current literature, policy documentation, and other publication outlets online. A second source examines optimal accreditation agency examples in-depth. Challenges and opportunities are discussed within the Zambian context, including recommendations on how to help overcome each challenge and capitalize on opportunities.
\end{abstract}

Keywords: Accreditation, Quality Assurance, Higher Education, Good Governance, Leadership, Strategic Planning

\section{Introduction}

Accreditation is among the most important areas of HE management worldwide. It is an area that focuses on quality assurance and the guiding principles of good governance. Each of the main HE organizational areas of strategy, structure, technology, and culture must consider accreditation to best meet the growing demands of stakeholders at all levels to ensure that graduates are able to perform at optimal levels that meet national and international standards of excellence.

And yet, too often HEIs fall short in their accreditation pursuits. For instance, many countries are just starting to establish national accreditation standards, and too often national standards do not always meet international standard requirements. The overall quality of HE is often measured based on the competencies of what students learn. This learning is essential if students are able to successfully transition into the workforce at local and national levels, as well as if they hope to find jobs in other country contexts. Thus mobility is another primary outcome indicator of a quality HE.

There are seven types of accrediting agencies: national (e.g., the Higher Education Authority in Zambia), international, regional, ${ }^{1}$ faith-related, career-related, programmatic (e.g., Accreditation Board for Engineering and Technology [ABET], Council for the

*Corresponding author email: stephensimukanga@ymail.com; †Corresponding author email: wjamesjacob@gmail.com.
Accreditation of Educator Preparation [CAEP], Carnegie Project on the Education Doctorate [CPED], etc.), and support agencies. Countries that tend to lead in accreditation often set national and international standards in various disciplines and fields. For instance, ABET sets international standards for excellence in engineering, applied science, and computing. Its headquarters is in Washington, DC, but works with HEIs worldwide, so long as they meet ABET accreditation criteria. Medical standards often differ from country to country, making it difficult for graduates of many medical disciplines to find employment in countries outside of the one they received their degree(s). Law is another locally-central discipline that often requires expertise and mastery of local and national laws that often differ in each country.

It is important to recognize and appreciate the great diversity that exists in HE worldwide. There is also no single cookie cutter or onesize-fits-all model of HE accreditation. Most accrediting agencies strive to provide a "shared understanding about the dimensions of quality that would be useful. These guiding principles are one effort to move toward such understanding while acknowledging and respecting the many differences of history, culture, beliefs and values that shape our systems of higher education and our perspectives on quality" (CIQG 2015).

International accrediting agencies include UNESCO, Organisation for Economic and Co-operative Development (OECD), ${ }^{2}$ the International Network of Quality Assurance Agencies in Higher Education (INQAAHE), ${ }^{3}$ Council for Higher Education Accreditation 
International Quality Group (CHEA/CIQG), the Arab Network for Quality Assurance in Higher Education (ANQAHE), the Asia-Pacific Quality Network (APQN), ${ }^{4}$ and the European Higher Education Area. ${ }^{5}$ Further new agencies have emerged in Africa - the African Quality Assurance Network (AfriQAN) and the East African Quality Assurance Network (EAQAN) - both aimed at ensuring improvement in the quality of African higher education.
There are four key principles of good governance that are essential in establishing and maintaining integrity throughout the quality assurance and accreditation processes. These include information flow, coordination, transparency, and accountability (Sutin and Jacob 2016). The most successful HE leaders ground their leadership on core values and characteristics as portrayed in Figure 1.

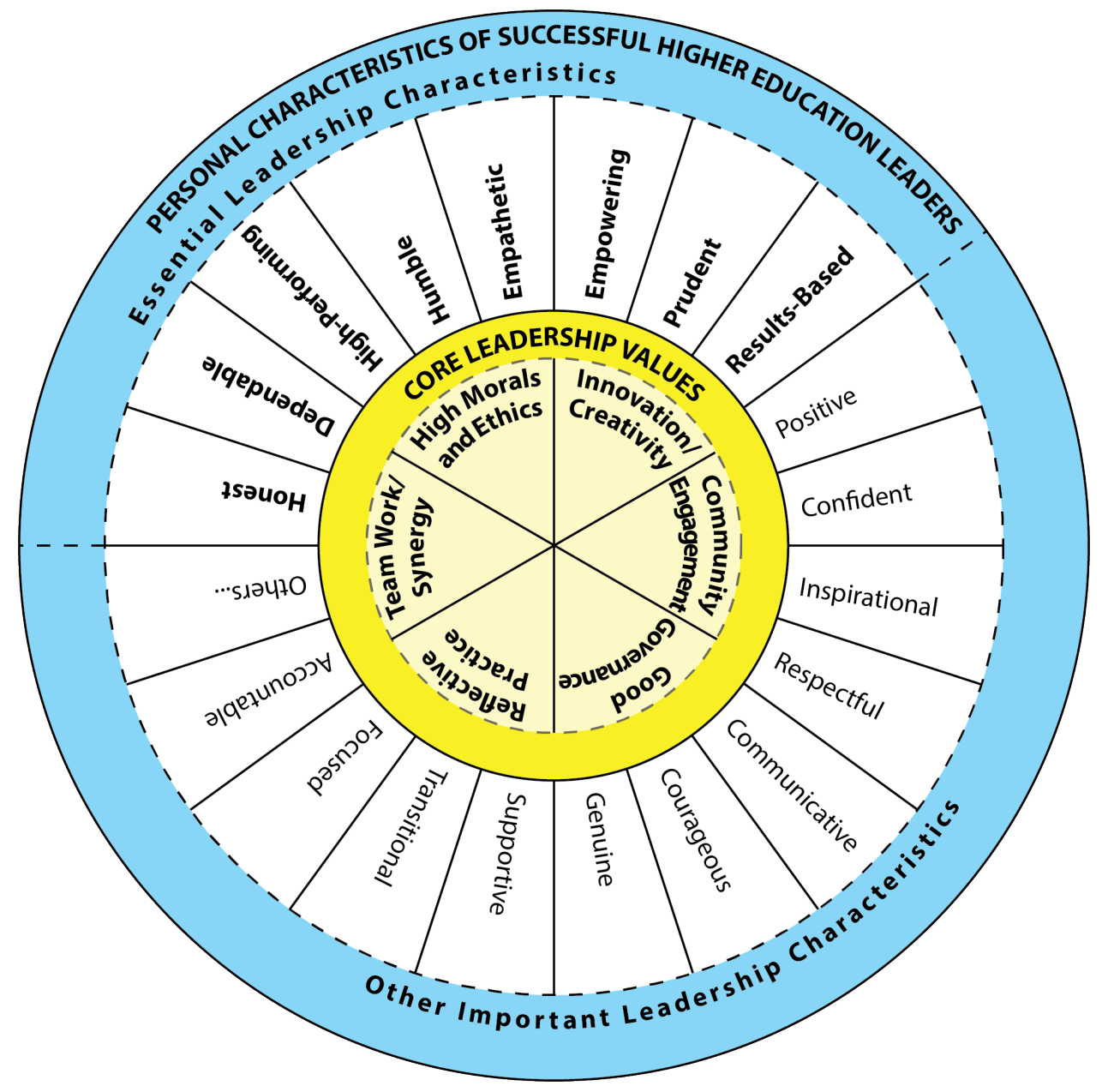

Figure 1. The Higher Education Leadership Wheel for Successful Accreditation Implementation

Source: Adapted from Stewart E. Sutin and W. James Jacob (2016, p. 2). 
In addition to the core leadership values outlined in Figure 1, CHEA (2016, p. 10) advocates that there are four additional core values specifically related to accreditation:

1. HEIs have the primary responsibility for academic quality: They are the leaders and the primary sources of authority in academic matters.

2. Institutional mission is central to all judgments of academic quality.

3. Institutional autonomy is essential to sustaining and enhancing academic quality.

4. Academic freedom flourishes only in an environment of academic leadership of institutions.

HE quality ensures that curriculum is designed to provide meaningful, competency-based education opportunities to students. Each HEI has the potential to achieve quality, excellence, and accreditation. Quality is also based on core values, ethics, and morals as Figure 1 portrays.

We need to avoid making unilateral decisions without first consulting others within the university which is like making decisions in a vacuum. HE administrators also need to be able to maintain national and international standards of excellence, identify and stop corruption, and have a strategic plan and ability to deal effectively and efficiently with inevitable changes.

In this paper we first provide an overview of the important leadership role the Higher Education Authority plays in providing accreditation and quality assurance guidance to HEIs in Zambia. We then provide an in-depth analysis of the strengths, weaknesses, opportunities, and challenges that HEIs face with accreditation in Zambia. We conclude with a list of five recommendations to help HE leaders better understand and prepare for sustainable quality in the accreditation process.

\section{The Higher Education Authority}

The Government of the Republic of Zambia has traditionally invested in primary and secondary education without con-commitment and development in tertiary education. So we have seen an increase in secondary school graduates without a corresponding increase in tertiary education as alluded to above. Because of this, there has been little access to HE opportunities for many of these graduates.

Realizing this outcome, the government introduced new legislation through the University Act No. 26 of 1992 which provided for participation of private HEIs in the higher education sector. The 1992 Act was repealed in 1999 and replaced with the University Act No. 11 of 1999 which further provided for participation of private
HEIs but introduced affiliation of colleges to public universities. The nation saw a proliferation of HEIs following the introduction of this legislation. At that time there was no established authority to oversee the quality of HEIs in the country. After opening up the HE sector to allow the private sector, there has been a rapid mushrooming of HEIs being established in the country.

In order to address issues of quality in the HEIs, the government repealed the University Act No. 11 of 1999 and replaced it with the Higher Education Act No. 4 of 2013. This legislation provided for the creation of the Higher Education Authority (HEA) whose mandate is to provide monitoring, evaluation, and quality standards to HE throughout the country. The commencement of this act was in August 2013. The Board of the HEA was instituted in October 2014. Effectively, the HEA came into operation at this time.

The work of HEA involves ensuring that an HEI meets set minimum criteria for registration as a private HEI. Further the HEA is also mandated to accredit learning programs in both public and private HEIs and also give technical assistance and capacity building to the government. In the Higher Education Act No. 4 there is a whole list of functions that the HEA is charged with, but this is a brief summary of its focus.

For the first year the HEA operated through an Interim Secretariat. Then, in January 2016, the first Director General was appointed, and this was followed by the appointment of the Director of Quality Assurance in August 2016.

The strategy for 2016 was to develop policies that HEIs could use in registration and accreditation and also to govern itself. After this, registration of private universities commenced. The next stage was the accreditation of learning programs in both public and private HEIs. In 2017, the HEA began auditing all Zambian HEIs by looking at what HEIs promised to deliver and to determine if they were on course or not. So, these are the three phases thus far-registration of private universities, accreditation of learning programs, and auditing of these HEIs. The HEA has registered 61 private HEIs as of December 2017. These HEIs comprise open, distance, and elearning $(\mathrm{ODeL})$ and face-to-face institutions. Approximately 21 of these HEIs are entirely focused on ODeL.

The next stage the HEA focused on was the development of policies both for managing the process and also for administering. In the beginning of November 2016, the accreditation process was publicized. All $61 \mathrm{HEIs,} \mathrm{including} \mathrm{the} \mathrm{six} \mathrm{public} \mathrm{universities,} \mathrm{that}$ were registered and the six public universities were informed about the accreditation criteria, which included a template for submitting to the HEA when they were ready.

The accreditation process will undoubtedly take longer than the registration process. This is somewhat of a new process in Zambia. Many HEIs have their university senates, and they have been offering HE courses for years. At this stage we have not established a 
required timeline for accreditation. We are hoping that by the end of 2017 there will be a good number of HEIs which have already started down the path toward achieving accreditation.

One of the key roles of the HEA is to follow the principles of good governance, including informing the public about the status of institutional and learning program accreditation. This will help inform future students, parents of students, and the community at large, which HEIs have accredited their programs. The public will be able to make their own decisions based on this information sharing and dissemination. The accredited programs will most likely continue to succeed, while those that don't merit accreditation status will eventually diminish. If programs are not accredited, student graduates will not be able to secure jobs or transfer to other universities because of their inability to articulate credits within Zambia and overseas.

The HEA has a unique relationship with the Zambian Qualifications Authority (ZAQA) by offering oversight of all universities in Zambia. There are four agencies which work with ZAQA and with the Zambia Qualifications Framework (ZQF) — the HEA; the Technical Education, Vocational and Entrepreneurship Training Authority (TEVETA) ${ }^{6}$ (e.g., technical colleges and trades training institutes); the Teaching Council (all colleges of education); and the Examinations Council of Zambia. The HEA oversees all universities and all HEIs other than those regulated by TEVETA and the Teaching Council. The HEA provides periodic reporting to ZAQA in reference to the accreditation processes of learning programs.

\section{Strengths of Accreditation in Zambia}

Prior to the establishment of the HEA, there was a significant amount of public distrust of the private HE sector. They would rather attend and support established public HEIs than private HEIs. Most private HEIs were looked down upon with suspicion. This process of $\mathrm{HE}$ accreditation process brings an assurance to the public that accredited learning programs are of the same quality, curriculum, and learning outcomes. While the instructors are undoubtedly different, accredited programs will bring a quality assurance to the public.

The Higher Education Act No. 4 of 2013 requires HEIs to become accredited by law. This is the mandate of the HEA. The HEA leadership has significant leadership capacity and an ability to provide guidance and technical assistance to help HEIs successfully navigate the $\mathrm{HE}$ accreditation process. A database has been established of approximately 300 content area experts in every major discipline and field of study, who will be involved in the reviewing and evaluation of the accreditation process in Zambia.

\section{Weaknesses in the Accreditation and Quality Assurance Processes}

Because it is a new phenomenon in Zambia, it will take time for HEIs to become accredited and meet national and international standards. While a database of content area experts and evaluators is available, they are not experienced in the review and evaluation processes. Most lack the general capacity and knowhow, but it is an area that we are committed to strengthen.

From the university's side, it is a new thing for most of their administrators to go through the accreditation process. There is a bit of resistance thus far, but it is hoped that this negative stereotype will be overcome. In addition to its role as an enforcement agency, the HEA primarily serves as a partner in the quality assurance and accreditation process.

\section{Opportunities in Reference to Zambian Accreditation}

The opportunities are there for HE administrators to be very proactive and become a leader in accreditation. It will provide their HEIs with positive public perception and they will be better able to attract students to their quality program offerings. The Zambian Qualifications Authority will help provide quality assurance for those learning programs and HEIs which meet the HEA registration and accreditation criteria.

ZAQA is part of the UNESCO Qualifications Network, and because of this, the ZAQA registered accreditation of learning programs will be recognized beyond Zambia.

Zambia is also fortunate to have a few neighboring HEIs in the sub-region of Africa, because ultimately there is a protocol for the SADC sub-region of transferability of credits, mobility of students and faculty. The Addis Ababa Regional Convention on the Recognition of Studies, Certificates, Diplomas, Degrees and other Academic Qualifications in Higher Education in the African States, adopted in Addis Ababa on 12 December 2014 is another protocol that Zambia is required to sign. This will reinforce the need for quality assurance mechanisms in the higher education sector. A Global Convention of recognition of qualifications under UNESCO is likely to be passed by UNESCO in 2019. Accreditation goals will help build greater collaboration capacity of HEIs with other HEIs in Africa and globally. 


\section{What Accreditation Challenges Does Zambia Currently Face?}

The HEA is a new organization and is still building up its capacity, skills, and technical assistance offerings. But, on the other side, because it is a rather new phenomenon, HEIs should be encouraged to get started. Most HEIs do not have their own quality assurance focal persons and units. This creates a challenge for most HEIs. They need to begin by appointing/hiring a quality assurance focal person and establish quality assurance units.

While many of our more established HEIs have senates that have operated for many years, they need to be trained and informed that part of their role is to ensure quality and accreditation of their learning programs. It is an ongoing challenge to help provide training to these focal persons and HE leaders.

The expectations from society are very high. In many cases there are some misunderstandings of what accreditation is. Some of the members of the public believe that one of the roles of the HEA is to close HEIs that do not meet accreditation. However, we understand this as an ongoing and learning process that will take time. Therefore, the HEA is required to go about it carefully and cautiously.

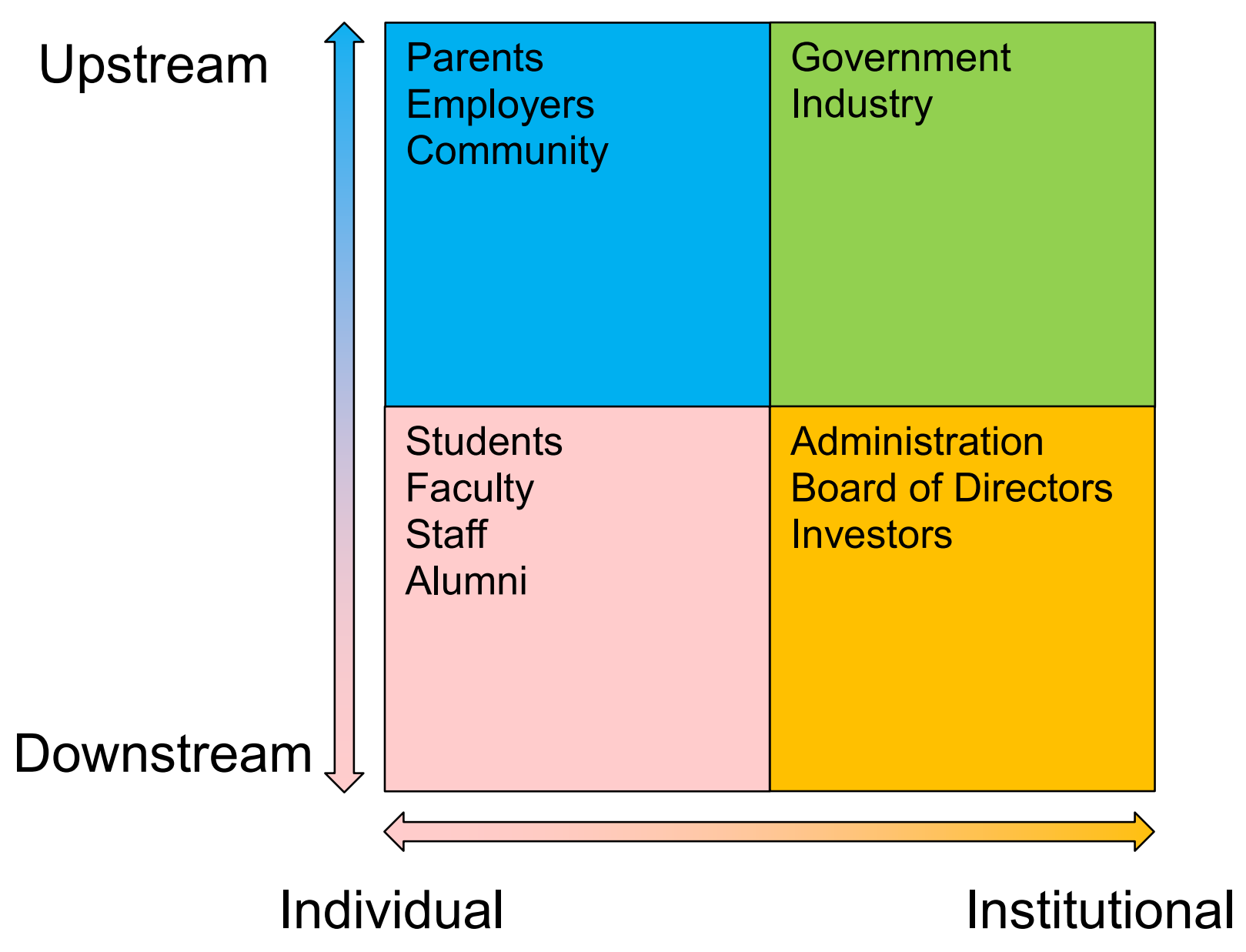

Figure 2. Corruption in Higher Education Matrix among Individual and Institutional Stakeholder Groups

Source: Created by the authors from UNESCO and CHEA/CIQG (2016, pp. 1-2). 
Corruption remains one of the most prevalent challenges in HE today. The HEA is required to take action and to take a positive stand against corruption in HE:

One reason is that in most domains university graduates will provide the future leadership of their communities and drive their development. If they experience corrupt behaviour as a normal part of their student days they are less likely to avoid such practices later in life. A doctor who was given police protection after exposing a massive cheating ring involving medical school entrance exams in India commented: 'The next generation of doctors is being taught to cheat and deceive even before they enter the classroom'. (UNESCO and CHEA/CIQG 2016, p. 2)

Graduates from HEIs are expected to be able to have core competencies in their respective fields and disciplines when entering the workforce. Corruption too often hinders students' ability to obtain these core competencies, and this ultimately erodes employer and public trust in our public and private HEIs. "Corruption in higher education has a high cost to society" (UNESCO and CHEA/CIQG 2016, p. 2).

Most Zambian HEIs are driven by their ability to generate revenues, rather than simply offering a public service. This opens the door and becomes fetter for corruption. The consequences for corruption - where student graduates are unable to perform at required industry and government standards - will ultimately prevent them from being able to secure jobs.

The auditing process will hopefully help offset and limit corruption among Zambian HEIs. This includes ensuring that incoming students are qualified to begin their HE studies. It also means that learning programs are providing sufficient learning outcomes such that graduates are able to perform at national and international standards of excellence.

\section{Conclusion and Recommendations}

In conclusion, we provide five recommendations on how to avoid common accreditation pitfalls. First, it is important to understand what is meant by accreditation. Begin by defining these key terms. Accreditation is not certification or licensure. According to Bogue and Bingham Hall (2003, p. 22) “'Accreditation' is often, and incorrectly, used interchangeably with 'certification' and 'licensure,"” and this highlights the complexity of the definition. It is almost impossible to improve or measure quality without first defining it.

Second, it is important to dispel false theories and definitions of accreditation. This in many cases takes a paradigm shift in the way
HE administrators and government planners think. Bogue and Bingham Hall (2003, p. 5) note how "Certain conventional assumptions about quality are often widely held" by stakeholders at all levels:

- Only high-cost universities have quality.

- Only large and comprehensive HEIs have quality.

- Only highly selective HEIs have quality.

- Only nationally recognized HEIs have quality.

- Only a few HEIs have quality.

- Only HEIs with impressive resources have quality.

Each of these are for the most part inaccurate depictions of quality assurance and accreditation. Another common error is that some stakeholders argue there can only be one (or at best a few) flagship university - usually the largest in the country and most often the research university. The reality is that no HEI can be completely comprehensive in any exhaustive sense - that is, have programs and services in every conceivable field of inquiry. Thus the word "comprehensive" will always have a limited meaning, and every HEI-no matter what its mission, its size, or its classification — will always have a limited mission.

Third, HE administrators need to be prepared. If done right, data collected through the quality assurance and accreditation processes should work to benefit administrators and sharing information with key stakeholder groups. If not, then resistance will be strong. It is essential to get the right leaders on board to implement necessary changes. With the right leadership in place sustainable change can be achieved. It is difficult, if not impossible, to achieve sustainable results without leadership buy-in, ownership, and support from key stakeholders throughout the strategic planning, implementation, and evaluation phases.

Fourth, HE leaders need to be involved in the definition and preparation efforts of accreditation. It is important to understand the many different stakeholder groups, such as:

- Students (future, current, and alumni)

- Faculty members

- Staff

- Administrators (at all levels, including boards of directors)

- Parents of Students

- Community members at local, regional, national, and international levels

- Industry (both public and private sectors)

- Government policy makers and planners

- Media

- Others... 
Stakeholder groups are part of the community engagement process that is fundamental in establishing a foundation of support and sustainable accreditation. Community engagement of stakeholders includes "sustainable networks, partnerships, communication media, and activities between HEIs and communities at local, national, regional, and international levels" (Jacob, et al. 2015, p. 1).

And finally, we support CIQG's (2015) seven international quality principles to help provide quality assurance to each of the key stakeholder groups listed above as well as ensuring that change initiatives meet national and international standards of excellence. These principles promote quality and (1) HE providers, (2) students, (3) society, (4) government, (5) accountability, (6) quality assurance and accreditation bodies, (7) sustained change.

\section{Notes}

1. The United States has six regional accrediting agencies, which are primarily based on geographic region: Higher Learning Commission (HLC), Middle States Association of Colleges and Schools (MSACS), New England Association of Schools and Colleges Commission on Institutions of Higher Education (NEASC-CIHE), Northwest Commission on Colleges and Universities (NWCCU), Southern Association of Colleges and Schools (SACS), and Western Association of Schools and Colleges (WASC-ACCJC) Accrediting Commission for Community and Junior Colleges; (WASC-ACSCU) Accrediting Commission for Senior Colleges and Universities.

2. These two multilateral agencies provide Guidelines for Quality Provision in Cross-Border Higher Education to UN- and OECDmember countries and for HEIs worldwide.

3. INQAAHE provides Guidelines of Good Practice for Quality Assurance for HEIs worldwide.

4. APQN offers higher education quality assurance for the Asia Pacific Region.

5. The European Higher Education Area has established the European Standards and Guidelines for Quality Assurance.

6. TEVETA is the regulatory agency of technical and vocational education and training (TVET) HEIs in Zambia.

\section{References}

Bogue, E. Grady, and Kimberely Bingham Hall. 2003. Quality and Accountability in Higher Education. Westport, CT: Praeger.

Council on Higher Education Accreditation (CHEA). 2016. A Board Member's Guide to Accreditation: The Basics, the Issues, the Challenges. Washington, DC: CHEA.
CHEA International Quality Group (CIQG). 2015. CHEA International Quality Group International Quality Principles. Washington, DC: CIQG.

Jacob, W. James, Stewart E. Sutin, John C. Weidman, and John L. Yeager. 2015. Community Engagement in Higher Education: Policy Reforms and Practice. Boston, MA: Sense Publishers.

Government of the Republic of Zambia. 2013. The Higher Education Act (No. 4 of 2013). Lusaka: Higher Education Authority, Ministry of Higher Education, Republic of Zambia.

Sutin, Stewart E., and W. James Jacob. 2016. Strategic Transformation of Higher Education: Challenges and Solutions in a Global Economy. Lanham, MD: Rowman \& Littlefield.

UNESCO and CHEA/CIQG. 2016. Combatting Corruption and Enhancing Integrity. Washington, DC: UNESCO-IIEP and CHEA/CIQG. 